Noch gewusst ...?

J. Gynäkol. Endokrinol. CH 2021 · 24:39-42 https://doi.org/10.1007/s41975-021-00180-5 Angenommen: 27. Januar 2021 Online publiziert: 18. Februar 2021 () Der/die Autor(en) 2021

\author{
Heike A. Bischoff-Ferrari ${ }^{1,2,3} \cdot$ Reto W. Kressig ${ }^{4,5}$. Christian Meier ${ }^{6,7} \cdot$ Petra Stute ${ }^{8,9}$ \\ 'Geriatrie, Universitätsspital Zürich, Zürich, Schweiz \\ ${ }^{2}$ Universitäre Klinik für Akutgeriatrie, Stadtspital Waid \& Triemli, Zürich, Schweiz \\ ${ }^{3}$ Lehrstuhl Geriatrie und Altersforschung, Universität Zürich, Zürich, Schweiz \\ ${ }^{4}$ Universitäre Altersmedizin FELIX PLATTER, Basel, Schweiz \\ ${ }^{5}$ Klinische Professur für Geriatrie, Universität Basel, Basel, Schweiz \\ ${ }^{6}$ Klinik für Endokrinologie, Diabetologie und Metabolismus, Universitätsspital Basel, Basel, Schweiz \\ ${ }^{7}$ Endokrinologische Praxis, Osteologisches Universitätsforschungszentrum DVO, Basel, Schweiz \\ ${ }^{8}$ Abteilung für Gynäkologische Endokrinologie und Reproduktionsmedizin, Frauenklinik, Inselspital, Bern, \\ Schweiz \\ ${ }^{9}$ Schweizerische Gesellschaft für Gynäkologische Endokrinologie und Menopause, SGEM, Lausanne \\ Schweiz
}

\title{
Empfehlung zu Vitamin D im Rahmen der COVID-19-Pandemie für Geriater*Innen und Hausärzt*Innen
}

\section{Ausgangslage}

In der aktuellen Pandemiesituation sind volksgesundheitliche Massnahmen zur Risikoverminderung der COVID-19-Infektion dringend gesucht. Die Hinweise zu Vitamin D als mögliche präventive Massnahme häufen sich und obgleich die in einer idealen Welt geforderte Evidenz einer grossen Studie zu Vitamin D bei COVID-19-erkrankten Patienten aktuell aussteht, sind aus einer volksgesundheitlichen Sicht in der aktuellen Krisensituation umsichtige Regeln, gestützt auf eine umfassende Risiko-Benefit-Analyse, notwendig, um potenziell Menschenleben zu retten. Aus dieser Perspektive betrachtet sprechen aus volksgesundheitlicher Sicht mehr Argumente für eine präventive Gabe von 400 bis 1000 IE Vitamin D am Tag im Rahmen der COVID-19-Pandemie als dagegen.

Dieses Statement basierend auf neusten wissenschaftlichen Daten soll die aktuelle Kontroverse zu Vitamin D in der Laienpresse sachlich ergänzen und Geriater ${ }^{\star} I n n e n$ und Hausärzt ${ }^{\star} I n n e n$ unterstützen, die Aussagen in der Laienpresse einzuordnen.

\section{Was ist die wichtigste Evidenz für eine Empfehlung zur Vitamin-D-Supplementation in der aktuellen COVID-19- Pandemie-Situation?}

Das wichtigste Argument für eine volksgesundheitliche Empfehlung zur präventiven Gabe von Vitamin D im Rahmen der aktuellen Pandemiesituation ist eine aktuelle Metaanalyse von 42 hochqualitativen klinischen Studien mit total 46.331 Studienteilnehmern und Studienteilnehmerinnen im Alter von 0 bis 95 Jahren. Ziel dieser Metaanalyse war herauszufinden, inwieweit eine Vitamin-D-Gabe das Risiko von akuten Atemwegsinfekten senken kann. Einschränkend bezogen aufdie aktuelle Pandemiesituation ginges hier um akute Atemwegsinfekte jeglicher Art, also nicht speziell um COVID-19verursachte akute Atemwegsinfekte. Das Resultat dieser Metaanalyse ist zudem noch nicht zur Publikation angenommen, aber bereits umfassend in einer Vorpublikation auf dem Internet einsehbar ${ }^{1}$.

\footnotetext{
1 Vorpublikation: https://www.medrxiv.org/ content/10.1101/2020.07.14.20152728v3.
}

Über alle 42 Interventionsstudien dieser Metaanalyse hinweg zeigt sich ein bescheidener Effekt mit einer signifikanten $9 \%$ igen Verminderung der akuten Atemwegsinfekte unter Vitamin D (Odds Ratio $[\mathrm{OR}]=0,91,95 \%$-KI 0,84-0,99). Die Autoren weisen jedoch darauf hin, dass die Analyse aller Studien möglicherweise den Benefit von Vitamin D unterschätzt, weil eine grosse Heterogenität zwischen den Studien festgestellt wurde. Tatsächlich zeigte das Ergebnis der Studien, welche die heutige Empfehlung von täglich 400-1000 IE Vitamin D untersucht hatten, ein deutlicheres und homogeneres Ergebnis, mit einer Verminderung der akuten Atemwegsinfekte um $30 \%(\mathrm{OR}=0,70,95 \%$-KI 0,55-0,89). Und für 8 Studien in der täglichen Dosierung von 400 bis 1000 IE Vitamin D in einer Anwendung bis zu 12 Monaten war das Ergebnis am ausgeprägtesten und homogensten mit einer Verminderung der akuten Atemwegsinfekte um $42 \%(\mathrm{OR}=0,58,95 \%$-KI 0,45-0,75).

Die wöchentlichen oder monatlichen Bolusgaben von Vitamin D waren hingegen nicht wirksam in der Verminderung akuter Atemwegsinfekte, während die tägliche Gabe von Vitamin D das Risi- 
ko akuter Atemwegsinfekte unabhängig von der Dosierung um $25 \%$ verminderte (OR=0,75, 95\%-KI 0,61-0,93).

Aktuell findet eine Ergänzung dieser neusten Metaanalyse mit den Daten der DO-HEALTH-Studie statt [1]. DOHEALTH untersuchte die zusätzliche Gabe von 2000 IE Vitamin D am Tag und zeigte eine signifikante $16 \%$ ige Verminderung jeglicher Infekte, jedoch nur bei den jüngeren Teilnehmern und Teilnehmern im Alter von 70 bis 74 Jahren. In DO-HEALTH hatte der überwiegende Teil der Studienteilnehmer ${ }^{\star}$ Innen zu Beginn der Studie keinen Vitamin-D-Mangel und alle Studienteilnehmer ${ }^{\star}$ Innen durften die heutige Empfehlung von 800 IE Vitamin D am Tag zusätzlich zur Studienmedikation einnehmen.

Zusammenfassend bezüglich der Einordnung dieser neusten Metaanalyse ist deren Ergebnis aus volksgesundheitlicher Sicht in der aktuellen Pandemiesituation relevant, weil COVID-19 ein akuter viraler Atemwegsinfekt ist. Mit und im schlimmsten Fall ohne Ansprechen auf das COVID-19-Virus ist eine Reduktion jeglicher akuten Atemwegsinfekte um $30 \%$ mit der Einnahme von 400 bis 1000 IE Vitamin D am Tag volksgesundheitlich hoch relevant. Im Rahmen einer Risiko-Benefit-Abwägung käme mit dieser Empfehlung zudem niemand zu Schaden, im Gegenteil, mit dieser Empfehlung könnten minimal andere virale und bakterielle akute Atemwegsinfekte gesenkt werden und vulnerable ältere Menschen mit Vitamin-D-Mangel hätten den belegten Benefit einer Risikoverminderung von Knochenbrüchen und Stürzen [2-6]. Bezüglich der Sicherheit ist Vitamin D in der täglichen Dosierung von 400 bis 1000 IE am Tag bereits in den präventiven Vitamin-D-Empfehlungen seitens des BAG (Federal Commission for Nutrition. Vitamin D deficiency: Evidence, safety, and recommendations for the Swiss Population. Expert report of the FCN.Zurich: Federal Office for Public Health, 2012) verankert, die zur Prävention ohne notwendige vorherige Testung des Blutspiegels zur Anwendung kommen, mit $97 \%$ Sicherheit den Vitamin-DMangel korrigieren und Menschen ohne Vitamin-D-Mangel nicht gefährden [7].

\section{Welche Hinweise gibt es spezifisch zu Vitamin D und COVID-19?}

Bezogen auf COVID-19 wurde in den vergangenen Monaten in der überwiegenden Anzahl publizierter Kohortenstudien beobachtet, dass Personen mit einem Vitamin-D-Mangel ein erhöhtes COVID-19-Risiko und eine erhöhte COVID-19-Mortalität aufweisen [8-12]. Kohortendaten belegen jedoch keine Kausalität und können wesentliche Schwächen aufweisen [13].

Dazu kommen basiswissenschaftliche Studien, die zeigen, dass Vitamin D den COVID-19-induzierten Zytokinsturm $\mathrm{zu}$ unterdrücken scheint $[14,15]$, als mögliche Erklärung, warum in verschiedenen Kohortenstudien ein höherer Vitamin-D-Blutspiegel mit einem geringeren Schweregrad der COVID-19Erkrankung korreliert [8-12]. Bekannt ist, dass Vitamin D eine Rolle in der angeborenen und akuten Immunantwort spielt [16]. Entsprechend ist der Vitamin-D-Rezeptor (Andockstelle) auf vielen Zellen des Immunsystems verankert [16] und vermittelt zum Beispiel die Freisetzung von Entzündungsfaktoren (Zytokinen) durch sogenannte Fresszellen und trägt damit zu einer Hochregulation von antimikrobiellen Substanzen bei, die eine antivirale Wirkung haben [17, 18]. Bezogen auf die Prävention akuter Atemwegsinfekte ist zudem bekannt, dass Virusinfektionen in der Lunge die Aktivierung von Vitamin $\mathrm{D}$ in dessen aktiver Form $(1,25$ Dihydroxyvitamin D) fördern und damit antivirale Abwehrmechanismen (Cathelicidin) in Gang kommen $[17,18]$.

Bezüglich Interventioneller Studien zur Rolle einer Vitamin-D-Therapie bei COVID-19-Patienten sind zwei kleine Pilotstudien publiziert:

1. In einer randomisierten Interventionsstudie mit Calcifediol (VitaminD-Metabolit) wurde eine signifikante Verringerung des Bedarfs an Intensivstationsbehandlungen bei 76 Krankenhauspatienten mit einer belegten COVID-19-Lungenentzündung festgestellt. Während in der Kontrollgruppe $50 \%$ intensivstati- onspflichtig wurden, waren es in der Calcifediolgruppe $2 \%$ [19].

2. In einer placebokontrollierten Interventionsstudie mit 40 COVIDpositiven Menschen mit keinen oder leichten Symptomen und einem belegten Vitamin-D-Mangel zeigte sich unter einer hoch dosierten VitaminD-Gabe, dass $63 \%$ der Teilnehmer in der Interventionsgruppe und nur $21 \%$ in der Kontrollgruppe vor Erreichen der 3. Woche COVID-RNAnegativ wurden [20].

Bisher fehlen große klinische Interventionsstudien, die die Rolle von Vitamin D bei COVID-19-erkrankten Menschen belegen. Eine große Studie bei COVID19-erkrankten Menschen läuft derzeit in Boston (VIVID: https://clinicaltrials. gov/ct2/show/NCT04536298).

\section{Stellen die ersten DO-HEALTH- Resultate die aktuellen Empfehlungen zu 800 IE Vitamin D am Tag bei Erwachsenen im Alter 60+ in der Prävention von Frakturen infrage?}

DO-HEALTH zeigte keinen Benefit von Vitamin D bezüglich des Risikos nichtvertebraler Frakturen. Wichtig ist jedoch, dass sich die Ergebnisse von DOHEALTH auf die zusätzliche Einnahme von 2000 IE Vitamin D am Tag (zusätzlich $\mathrm{zu}$ der aktuellen Empfehlung von 800 IE am Tag) bei aktiven und gesunden älteren Menschen im Alter 70 und älter beziehen, die im überwiegenden Teil keinen Vitamin-D-Mangel hatten und in über $30 \%$ der Fälle während der Studie 800 IE am Tag zusätzlich zur Studienmedikation einnahmen. Damit widersprechen die DO-HEALTH-Ergebnisse den aktuellen Empfehlungen von 800 IE Vitamin D am Tag für ältere Erwachsene nicht und stellen auch nicht die belegte Wirkung von 800 IE Vitamin D am Tag in der Prävention von Stürzen \& Frakturen bei vulnerablen älteren Menschen mit Vitamin-D-Mangel und Osteoporose infrage [2-6].

Als wichtige Übereinstimmung bezüglich Vitamin-D-Empfehlungen für die Knochenbruchprävention, Sturz- 
prävention und Prävention von akuten Atemwegsinfekten sollten Bolusgaben von Vitamin D vermieden werden und die tägliche Einnahme von Vitamin D bevorzugt stattfinden $[5,6]$.

Zusammenfassend fehlt bezüglich Vitamin D zum heutigen Zeitpunkt zwar der abschliessende Beweis einer grossen klinischen Studie bei COVID-19-erkrankten Patienten, jedoch sollte in der aktuellen Pandemiesituation die Evidenz bezogen auf die Senkung jeglicher akuter Atemwegsinfekte um $30 \%$ mit der seitens des BAG seit 2012 bestehenden präventiven Empfehlung von 400 bis 1000 IE am Tag (bezogen auf die Knochengesundheit) eine ausreichende volksgesundheitliche Basis darstellen, um jetzt $\mathrm{zu}$ reagieren. Alternativ werden, bis die Studienergebnisse der Boston-VIVID-Studie vorliegen, Monate vergehen, in denen die sichere und günstige und breit verfügbare präventive Massnahme von 400 bis 1000 IE Vitamin D am Tag bereits greifen könnte. Dazu befinden wir uns in der Wintersaison, in der mindestens jeder zweite erwachsene Mensch in der Schweiz einen Vitamin-D-Mangel hat [21] und die COVID-19-Hochrisikopopulation von vulnerablen älteren Menschen das höchste Risiko für einen Vitamin-D-Mangel trägt [22].

\section{Empfehlung}

1. Anhand dieser Risiko-Benefit-Abwägung in der aktuellen Pandemiesituation empfiehlt dieses ExpertenStatement allen Menschen ab 60 Jahren täglich 800 IE Vitamin D nicht nur bezogen auf die Knochengesundheit, sondern in der aktuellen Pandemiesituation auch zur Prävention akuter Atemwegsinfekte. Diese Empfehlung bezieht sich prioritär auf die neuste Metaanalyse mit 42 Studien, welche für die tägliche Dosis von 400 bis 1000 IE Vitamin D eine Verminderung akuter Atemwegsinfekte um $30 \%$ belegen konnte. Hausärzte sollten ihre Patienten jedoch darauf hinweisen, dass es bisher keinen Beleg gibt, dass Vitamin D auch vor akuten Atemwegsinfekten durch COVID-19 schützt, dass es jedoch zunehmen- de wissenschaftliche Hinweise gibt, dass die Behebung eines Vitamin-

D-Mangels das Risiko von schweren Krankheitsverläufen der COVID-

19-Atemwegsinfektion vermindern könnte.

Konsistent mit den 2012 publizierten Empfehlungen des BAG bedarf die Supplementation mit 800 IE Vitamin D am Tag keiner vorherigen Testung des Blutspiegels, weil diese Dosierung auch bei Menschen ohne Mangel sicher ist und in $97 \%$ der Fälle den Vitamin-D-Mangel sicher behebt.

2. Die Empfehlung von 800 IE Vitamin D am Tag sollte bei Menschen im Alter 60+ auch nach einer COVID19-Impfung fortgeführt werden zur Prävention jeglicher akuten Atemwegsinfekte.

Aus volksgesundheitlicher Sicht einer entsprechenden Benefit-Risiko-Betrachtung erscheint es in der aktuellen und eskalierenden Pandemiesituation wichtig, diese Präventionsempfehlung zur Senkung des Risikos für akute Atemwegsinfekte schnell umzusetzen.

Zitat Editorial Lancet 1-2021 zu Vitamin D und COVID-19: „Additional evidence could come in just too late. In an ideal world, all health decisions would be made based on overwhelming evidence, but a time of crisis may call for a slightly different set of rules."

\section{Korrespondenzadresse

Prof. Dr. med. Petra Stute
Abteilung für Gynäkologische
Endokrinologie und
Reproduktionsmedizin,
Frauenklinik, Inselspital
Friedbühlstrasse 19,
3010 Bern, Schweiz
petra.stute@insel.ch

Funding. Open access funding provided by University of Bern

\section{Einhaltung ethischer Richtlinien}

Interessenkonflikt. H.A. Bischoff-Ferrari, R.W. Kres sig, C. Meier und P. Stute geben an, dass kein Interessenkonflikt besteht.
Für diesen Beitrag wurden von den Autoren keine Studien an Menschen oder Tieren durchgeführt. Für die aufgeführten Studien gelten die jeweils dort angegebenen ethischen Richtlinien.

Open Access. Dieser Artikel wird unter der Creative Commons Namensnennung 4.0 International Lizenz veröffentlicht, welche die Nutzung, Vervielfältigung, Bearbeitung, Verbreitung und Wiedergabe in jeglichem Medium und Format erlaubt, sofern Sie den/die ursprünglichen Autor(en) und die Quelle ordnungsgemäß nennen, einen Link zur Creative Commons Lizenz beifügen und angeben, ob Änderungen vorgenommen wurden.

Die in diesem Artikel enthaltenen Bilder und sonstiges Drittmaterial unterliegen ebenfalls der genannten Creative Commons Lizenz, sofern sich aus der Abbildungslegende nichts anderes ergibt. Sofern das betreffende Material nicht unter der genannten Creative Commons Lizenz steht und die betreffende Handlung nicht nach gesetzlichen Vorschriften erlaubt ist, ist für die oben aufgeführten Weiterverwendungen des Materials die Einwilligung des jeweiligen Rechteinhabers einzuholen.

Weitere Details zur Lizenz entnehmen Sie bitte der Lizenzinformation auf http://creativecommons.org/ licenses/by/4.0/deed.de.

\section{Literatur}

1. Bischoff-Ferrari HA, Vellas B, Rizzoli R et al (2020) Effect of vitamin D supplementation, omega-3 fatty acid supplementation, or a strength-training exercise program on clinical outcomes in older adults: the DO-HEALTH randomized clinical trial. JAMA 324(18):1855-1868

2. Bischoff-Ferrari HA, Orav EJ, Willett WC et al (2012) A pooled analysis of vitamin $D$ dose requirements for fracture prevention. N Engl J Med 367:40-49

3. Bischoff-Ferrari HA, Dawson-Hughes B, Staehelin HB et al (2009) Fall prevention with supplemental and active forms of vitamin D: a meta-analysis of randomised controlled trials. BMJ 339:b3692

4. Bischoff-Ferrari HA, Orav EJ, Abderhalden $L$, Dawson-Hughes B, Willett WC (2019) Vitamin D supplementation and musculoskeletal health Lancet Diabetes Endocrinol 7(2):85

5. Bischoff-Ferrari HA (2020) Hype about vitamin D substitution: what remains? Internist 61(11):1196-1203

6. Bischoff-Ferrari HA (2020) Vitamin D in geriatric patients. Internist 61(6):535-540

7. Bischoff-Ferrari HA, Shao A, Dawson-Hughes B, Hathcock J, Giovannucci E, Willett WC (2010) Benefit-risk assessment of vitamin $D$ supplementation. Osteoporos Int 21(7):1121-1132

8. Ilie PC, Stefanescu S, Smith L (2020) The role of vitamin $D$ in the prevention of coronavirus disease 2019 infection and mortality. Aging Clin Exp Res 32(7):1195-1198

9. Meltzer DO, Best TJ, Zhang H, Vokes T, Arora V, Solway J (2020) Association of vitamin D status and other clinical characteristics with COVID-19 test results. JAMA Netw Open 3(9):e2019722

10. Merzon E, Tworowski D, Gorohovski A et al (2020) Low plasma $25(\mathrm{OH})$ vitamin D level is associated with increased risk of COVID-19 infection: an Israeli population-based study. FEBS J 287(17):3693-3702 
11. Ali N (2020) Role of vitamin D in preventing of COVID-19 infection, progression and severity. JInfect Public Health 13(10):1373-1380

12. Munshi R, Hussein MH, Toraih EA et al (2021) Vitamin D insufficiency as a potential culprit in critical COVID-19 patients. J Med Virol 93(2):733-740

13. Rubin R (2021) Sorting out whether vitamin D deficiency raises COVID-19 risk. JAMA 325(4):329-330

14. Ahmed F (2020) A network-based analysis reveals the mechanism underlying vitamin $D$ in suppressing cytokine storm and virus in SARScoV-2 infection. Front Immunol 11:590459

15. Daneshkhah A, Agrawal V, Eshein A, Subramanian H, Roy HK, Backman V (2020) Evidence for possible association of vitamin $D$ status with cytokine storm and unregulated inflammation in COVID-19 patients. Aging Clin Exp Res 32(10):2141-2158

16. Bouillon R, Bischoff-Ferrari H, Willett W (2008) Vitamin $D$ and health: perspectives from mice and man. JBone Miner Res 23(7):974-979

17. Hughes DA, Norton R (2009) Vitamin D and respiratory health. Clin Exp Immunol 158(1):20-25

18. Vargas Buonfiglio LG, Cano M, Pezzulo AA et al (2017) Effect of vitamin D3 on the antimicrobial activity of human airway surface liquid: preliminary results of a randomised placebo-controlled double-blind trial. BMJOpen Respir Res 4(1):e211

19. Entrenas Castillo M, Entrenas Costa LM, Vaquero Barrios JMetal (2020) Effect of calcifediol treatment and best available therapy versus best available therapy on intensive care unit admission and mortality among patients hospitalized for COVID19: A pilot randomized clinical study. J Steroid Biochem Mol Biol 203:105751

20. Rastogi A, Bhansali A, Khare N et al (2020) Short term, high-dose vitamin $D$ supplementation for COVID-19 disease: a randomised, placebo-controlled, study (SHADE study). Postgrad Med J. https://doi.org/10.1136/postgradmedj2020-139065

21. Guessous I, Dudler V, Glatz N et al (2012) Vitamin D levels and associated factors: a population-based study in Switzerland. Swiss Med Wkly 142:w13719

22. Bischoff-Ferrari HA, Can U, Staehelin HB et al (2008) Severe vitamin D deficiency in Swiss hip fracture patients. Bone 42(3):597-602

Hinweis des Verlags. Der Verlag bleibt in Hinblick auf geografische Zuordnungen und Gebietsbezeichnungen in veröffentlichten Karten und Institutsadressen neutral.

\section{Teichmann, Gero (Hrsg.)}

\section{Operationsberichte Gynäkologie und Geburtshilfe}

Berlin Heidelberg: Springer-Verlag 2020, 1. Auflage, 249 S., 112 Abb., (ISBN: 978-3662-61426-6), 45,83 EUR

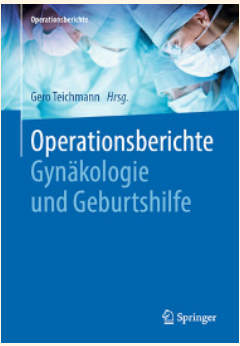

Speziell junge

Kolleginnen und

Kollegen sehen sich

manchmal gerade in der Anfangszeit der

Facharztausbildung gefordert, wenn nicht gar überfordert, nach erstmals

selbst durchgeführten Eingriffen einen qualitativ adäquaten Operationsbericht zu erstellen.

Neben der reichlich anfallenden administrativen Tätigkeit, die neben der klinischen Arbeit an der Patientin im Arbeitsalltag untergebracht werden muss, bleibt häufig zu wenig Zeit, sich über die Erstellung eines medizinisch und auch juristisch exakt formulierten Berichts ausreichend Gedanken zu machen. In manchen Abteilungen liegen für die am häufigsten vorkommenden Eingriffe mehr oder weniger strukturiert Muster-Operationsberichte vor, die von einer Facharzt- bzw. Oberarztgeneration zur nächsten entsprechend weitergegeben werden. Dass sich über dieses Verfahren Fehler einschleichen oder fortsetzen können, liegt in der Natur der Sache. Außerdem entwickeln sich die operative Technik und apparative Ausrüstung ständig weiter, so dass neue Verfahren und Operationsschritte in den OP-Bericht implementiert werden müssen.

In dem nun vorliegenden Buch „Operationsberichte Gynäkologie und Geburtshilfe", das in der bereits für andere Fachbereiche bewährten Reihe "Operationsberichte" erscheint, ist es in hervorragender Weise gelungen, den jungen Operateuren eine umfassende Vorlage für die vielfältigen Eingriffe unseres Faches zur Hand zu geben.
In einem Anfangsteil finden sich Ausführungen zu den unbedingt erforderlichen Basisdaten für einen angemessenen OPBericht. Dabei wird besonders auf die zweifelsfreie Zuordnung des OP-Berichts zu einer spezifischen Patientin hingewiesen. Auch die kurze Anamnese mit aktueller Symptomatik, Begleitmedikationen sowie die Indikationsstellung werden als unbedingt notwendige Angaben im OP-Bericht erwähnt. Vor allem wird darauf hingewiesen, exakte Größenangaben zu verwenden und nicht auf die häufig verwendeten Vergleiche wie frauenfaustgroß, knapp hühnereigroß oder handtellergroß zurückzugreifen. Es wird mit Recht darauf verwiesen, dass derartige Angaben heute als obsolet anzusehen sind.

Die außerordentlich große Anzahl an sinnvollen Vorlagen wird es den künftigen Operateuren enorm erleichtern, von Beginn der Ausbildung an sowohl fachlich als auch juristisch einwandfreie OP-Berichte zu erstellen. Wenn dabei nicht vergessen wird, die Berichte dem intraoperativen Verlauf entsprechend individuell zu modifizieren, wird dem vorliegenden Buch eine weite Verbreitung sicher sein.

\section{T. Fehm (Düsseldorf)}

